\title{
La validità della tecnica buttonhole nell'autopuntura
}

\section{Monica Spina}

\author{
U.O. Nefrologia e Dialisi, Ospedale N.S. di Bonaria, ASL 6, Sanluri (CA)
}

THE FEASIBILITY OF THE BUTTON-HOLE TECHNIQUE FOR SELF-CANNULATION

Abstract. The button-hole (BH) is a well-known alternative technique of venipuncture of arteriovenous fistulas (AVF). In our dialytic centers (U.O. of San Gavino and Center of Serramanna) we treated 42 patients using this technique from 2009 until today. At first we only used it in difficult or aneurysmatic AVF, but we soon extended the indication to all the AVFs of new formation; moreover, we selected a group of young and motivated patients for self-puncture and cannulation. In this last group of patients and in those of the Serramanna's Center, we obtained the best results in terms of reduction or absence of complications, especially infections. The BH, much more than the "rope-ladder" or the "area" techniques of venipuncture, needs a definite protocol and a strict follow-up. Being evident that pain and hemostatic time are sharply reduced by $\mathrm{BH}$, we believe that it is worthy or unique when self-cannulation and home treatment are programmed.

Key words: Buttonhole tecnique, Self-cannulation of AVF

Conflict of interest: None.

Financial support: None.

Ricevuto: 29 Gennaio 2014

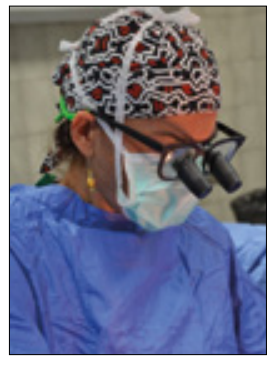

Monica Spina
La tecnica di venipuntura della FAV definita "a occhiello" o "buttonhole technique" $(\mathrm{BH})$ è ormai da tempo menzionata e sperimentata come tecnica alternativa a quella "ad area" o "a scala di corda". Twardowsky, già quarant'anni fa, la consigliava per quei casi di FAV tortuosa o corta, difficile da pungere a causa degli spazi ridotti per l'ago infissione (1).

Nel Centro Dialisi dove lavoro, tutte e tre le metodiche sono state e sono tuttora applicate; ogni tecnica di venipuntura presenta le proprie criticità che, direttamente o indirettamente, vengono a coinvolgere il paziente e il personale infermieristico della sala dialisi.

La scelta di una tecnica di venipuntura rispetto a un'altra, oltre che dai requisiti clinici del paziente, dipende anche dalle problematiche quotidiane di tipo organizzativo che coinvolgono il personale medico-infermieristico dedito alla cura dell'accesso vascolare.

Infatti, la tecnica $\mathrm{BH}$, a differenza delle altre tecniche di venipuntura, richiede un protocollo di procedura e una costante e vigile sorveglianza nel tempo.

Considerare la tipologia di paziente, la sua aspettativa di vita e, soprattutto, l'albero vascolare che mette a disposizione per la creazione di una FAV è stato per noi il punto di inizio per sperimentare la tecnica BH nella U.O. dell'Ospedale di San
Gavino e nel Centro Dialisi Decentrato (CAD) di Serramanna. Dal 2009 a oggi, abbiamo applicato la tecnica BH su 42 pazienti, riservandola, all'inizio, solo alle FAV aneurismatiche o con tratti da pungere difficili e profondi. Successivamente, abbiamo esteso la tecnica anche alle FAV giovani, di nuovo allestimento, raggiungendo i nostri obiettivi di salvaguardia dell'accesso vascolare, individuati come riduzione degli ematomi perivascolari da errata puntura, degli pseudo aneurismi, delle stenosi e delle dilatazioni aneurismatiche, complicanze comuni della tecnica di venipuntura a scala di corda e ad area (2).

Avendo sperimentato che la tecnica $\mathrm{BH}$ riduce anche la sensazione di dolore all'ago infissione e i tempi di emostasi dopo la rimozione degli aghi fistola, abbiamo volutamente proposto e insegnato questa tecnica ai pazienti giovani e motivati, in modo da coinvolgerli direttamente nelle manovre di autopuntura e di attacco e stacco al rene artificiale. Attualmente, nei nostri Centri Dialisi, 6 pazienti attuano l'autopuntura con la tecnica $\mathrm{BH}$ e autogestiscono la seduta emodialitica prescritta; sino a oggi, nessuno di questi pazienti ha presentato alcuna complicanza legata a questa metodica, anzi abbiamo osservato che questa metodica migliora l'empatia del paziente nei confronti della propria malattia e della FAV e la sua collaborazione nella gestione del lavoro in sala dialisi.

La letteratura evidenzia già da anni che l'infezione è una delle più temute complicanze della tecnica $\mathrm{BH}$; questa può localizzarsi solo al sito costante od occhiello oppure può diventare sistemica per la colonizzazione ematica dei comuni germi sa- 
profiti della cute, vedi lo stafilococco epidermidis (3).

Per esperienza acquisita, ho capito che il punto fondamentale si focalizza proprio sulla corretta procedura di rimozione delle croste che si formano sull'occhiello, sulle norme igieniche che l'infermiere e il paziente devono applicare in questa tecnica e sul corretto utilizzo dei dispositivi in uso (dispositivo "leva croste", aghi taglienti e aghi smussi).

Per ottenere dei risultati occorre che il personale di sala e il paziente siano collaborativi e propensi a cambiare abitudini, a non essere impazienti e frettolosi, a imparare e a rispettare un protocollo di procedura.

Gli ultimi studi di controllo sulle infezioni da tecnica $\mathrm{BH}$ in confronto con la tecnica a scala di corda (4-6) ci dicono che il problema è strettamente legato a questi aspetti procedurali, responsabili dell'insorgenza delle complicanze infettive locali e sistemiche.

Leggendo le esperienze di vari Centri Dialisi, italiani e di tutto il mondo, ognuno ha da dire la sua sui "pro e contro" della tecnica $\mathrm{BH}(7,8)$; se la tecnica $\mathrm{BH}$ nelle intenzioni originarie era nata per indicazioni selezionate e non per l'impiego generalizzato in tutti i pazienti in dialisi con FAV nativa, in base ai dati presenti in letteratura questo approccio sembra ancora valido. Nella nostra esperienza, i risultati migliori si sono avuti con $\mathrm{i}$ pazienti che si autopungono e in quelli che sono dializzati nel CAD di Serramanna. Infatti, nel CAD, il turnover degli infermieri è inferiore e il carico di lavoro è meno gravoso rispetto a quello dell'U.O. di San Gavino; questo ha permesso di dedicare più attenzione alla tecnica $\mathrm{BH}$ e di estenderla a quasi tutti i pazienti che vi sono dializzati. L'acquisizione di una tecnica di venipuntura che viene effettuata sempre dalla stessa mano con il tempo si perfeziona e annulla le complicanze.

Sono, quindi, d'accordo con il presupposto che la tecnica $\mathrm{BH}$ debba essere considerata come un'alternativa valida di venipuntura per i pazienti portatori di una FAV nativa; è utopistico poter pensare di applicarla a tutti i pazienti di un Centro Dialisi, ma, di sicuro, rappresenta un'alternativa valida o, anche, unica, soprattutto per coloro che hanno in programma un progetto di emodialisi autogestita e/o domiciliare.

\section{Riassunto}

La tecnica buttonhole è da tempo sperimentata come tecnica alternativa di venipuntura delle FAV native. Dal 2009 a oggi, nel Centro Dialisi di San Gavino e nel CAD di Serramanna, 42 pazienti sono stati sottoposti a questa tecnica, che era riservata, all'inizio, solo alle FAV difficili da pungere e aneurismatiche.

Successivamente, la tecnica è stata estesa anche alle FAV di nuovo allestimento e volutamente abbiamo motivato $\mathrm{i}$ pazienti giovani a utilizzarla per l'autopuntura. I risultati migliori, in termini di complicanze infettive, si sono avuti con i pazienti che si autopungono e in quelli che sono dializzati nel CAD. La tecnica buttonhole, a differenza delle tecniche di venipuntura a scala di corda e ad area, richiede un protocollo di procedura e la costante e vigile sorveglianza nel tempo.

Avendo sperimentato che la tecnica $\mathrm{BH}$ riduce la sensazione del dolore all'ago infissione e i tempi di emostasi, essa può essere considerata una tecnica di venipuntura valida o unica, soprattutto per i pazienti che hanno un programma di dialisi autogestita e/o domiciliare.

Parole chiave: Tecnica buttonhole, Autopuntura della FAV

Dichiarazione di conflitto di interessi: L'Autore dichiara di non avere conflitto di interesse.

Contributi economici agli Autori: L'Autore dichiara di non aver ricevuto sponsorizzazioni economiche per la preparazione dell'articolo.

\author{
Indirizzo dell'Autore: \\ Dr.ssa Monica Spina \\ U.O. Nefrologia e Dialisi \\ P.O. Nostra Signora di Bonaria \\ Via Roma 1 \\ 09037 San Gavino Monreale (CA) \\ mspina@aslsanluri.it
}

\section{Bibliografia}

1. Twardowsky Z, Kubara H. Different sites versus constant site of needles insertion into arterovenous fistola for treatment by repeted dialisi. Dial Transplant 1979; 8: 978-80.

2. Pergolotti A, Rich E, Lock K. The effect of the buttonhole method vs. the traditional method of AV fistula cannulation on hemostasis, needle stick pain, pre-needle stick anxiety, and presence of aneurysms in ambulatory patients on hemodialysis. Nephrol Nurs J. 2011; 38(4): 333-6.

3. Nesrallah GE, Cuerden M, Wong JH, Pierratos A. Staphylococcus aureus bacteremia and buttonhole cannulation:longterm safety and efficacy of mupirocin prophylaxis. Clin J Am Soc Nephrol. 2010;5(6):1047-53.

4. Grudzinski A, Mendelssohn D, Pierratos A, Nesrallah G.A.
Sistematic review of buttonhole cannulation practices and outcomes. Semindial. 2013; 26(4): 465-75.

5. Van Eps CL, Jones M, Ng T,et al. The impact of extendedhours home hemodialysis and buttonhole cannulation technique on hospitalization rates for septic events related to dialysis access. Hemodial Int. 2010 Oct; 14(4): 451-63.

6. Labriola L,Jadoul M. Infectious complications following conversion to buttonhole cannulation. Clin Nephrol. 2011; 76(5): 423

7. Casi Clinici. Giornale di Tecniche Nefrologiche e Dialitiche Anno XX; n.1/2 gennaio- giugno 2008.

8. Chow J, Rayment G, San Miguel S, Gilbert M. A randomized controlled trial of buttonhole cannulation for the prevention of fistula access complication.J Ren Care. 2011;37(2):85-93. 